\title{
CSO BASED ENERGY EFFICIENT CLUSTER PROTOCOL FOR WIRELESS SENSOR NETWORKS
}

\author{
D. Chandirasekaran and T. Jayabarathi \\ School of Electrical Engineering, Vellore Institute of Technology, Vellore, India
}

\begin{abstract}
In wireless sensor networks (WSNs) energy saving has become one of the prime optimization problem and clustering technique has been considered as most efficient techniques to achieve the same. The selection of cluster heads (CHs) plays a vital role in hierarchical based WSNs as it consume more energy owing to its additional duty of receiving, aggregating the data from the cluster member nodes and transmitting the same to the base station $(B S)$. Improper selection of CHs causes impact on network life time. In order to have an energy efficient network a suitable optimization algorithm is to be adopted to select the CHs. We propose a cluster protocol based on Cat Swarm Optimization (CSO) algorithm to reduce the energy consumption during cluster setup phase and transmission phase. The CSO cluster protocol is developed by considering intra-cluster distance of nodes to $\mathrm{CH}$ and residual energy of cluster member nodes. The algorithm is tested extensively on various scenarios of WSNs, varying number of sensor nodes and the CHs. The energy efficient scheme of proposed CSO performance is compared with other well-known protocols such as Low Energy Adaptive Clustering Hierarchy -Centralized (LEACHC) and Particle Swarm Optimization (PSO) based protocol to prove the superiority of it.
\end{abstract}

Keywords:

WSN, Cluster Head, Sensor Nodes, PSO, CSO

\section{INTRODUCTION}

Wireless Sensor Networks are distributed networks consist of sensor nodes (SNs) which can sense the indented information for what it has been deployed for and update the data to the base station [1]. WSN technology emerged in all areas of applications including medical, military, process and offshore industries due to its easy implementation and maintenance. However, the major issues with WSN are the deployment of the nodes, location awareness and energy aware clustering. In WSN, grouping the sensor nodes called clustering which is one of the most popular method employed to preserve the energy of the sensor nodes. To make clusters, the network is divided into several groups and each cluster has a leader called as cluster head $(\mathrm{CH})$. The role of $\mathrm{CHs}$ is to collect the sensed information from their member SNs within the clusters group, aggregate and transmit it to the BS. The cluster based routing protocols are popular techniques in terms of efficient communication and scalability [2]. In WSNs, the concept of cluster based routing protocol also efficiently used for energy saving in networks. The sensor nodes are grouped to form cluster with the help of data aggregation and fusion techniques at the right cluster head, which makes sure that the useful sensed data reaching the base station and thus energy reduction. Also the intra cluster communication helps in reducing the distance of sensor nodes present in a cluster, thus energy consumption is reduced. Naturally inspired algorithms provide better optimization solution in recent days, specifically for the WSN applications such as cluster based routing protocol as shown in Fig.1. A novel bio inspired optimization algorithm, called Cat Swarm Optimization (CSO) algorithm is reformed to integrate in to cluster based routing protocol in order to achieve a better network arrangement. The CSO protocol is evaluated in simulation and then validated using experimental-hardware test bed.

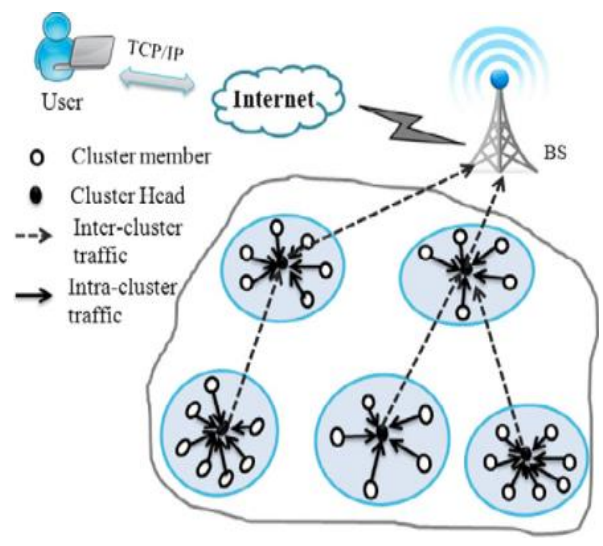

Fig.1. Cluster based protocol for WSNs

\section{REVIEW OF RELATED WORK}

A large number of clustering algorithms have been developed for WSNs. We present the review of related work based on both heuristic and bio inspired approaches. However, in this paper we focused more on bio inspired algorithms. Many Heuristic approaches based clustering algorithms [3-6] have been developed for WSNs. Among these LEACH [3] is a well-known clustering algorithm where the SNs elect themselves as a $\mathrm{CH}$ based on some probability. LEACH offers considerable energy saving and extension of network lifetime compared to minimum transmission energy (MTE) protocol. The disadvantage of LEACH algorithm is that the selection of $\mathrm{CH}$ could be possible with and SN with very low energy, which probably dies quickly and thus, shortens the life of the network. In order to overcome the issue with LEACH, HEED [7] and PEGASIS [8] algorithms have been developed. In PEGASIS, the SNs are formed as chain so that each SN transmits and receives the data only from its neighbour nodes. The $\mathrm{CH}$ is selected from the chain of SNs in each round of operation. PEGASIS is comparatively energy efficient than $\mathrm{LEACH}$, however it is unstable for large size networks. To extend the life time of the network, many algorithms [9-12] have been developed in recent past for WSNs cluster protocol. TL-LEACH [13], have been proposed by introducing a different level of hierarchy to improve the network lifetime over LEACH. However, the selection of secondary $\mathrm{CH}$ and non $\mathrm{CH}$ has been done based on intra cluster distance, which may cause energy imbalance to the network. M-LEACH algorithm [14] is proposed, to transmit the sensed data to the $\mathrm{CH}$ instead of direct 
communication with BS which saves energy compared to LEACH and TL-LEACH. However, the cluster set up phase is not and multi-hop data transfer between $\mathrm{CHs}$ is not considered. VLEACH protocol has been proposed in [15] in which some trim $\mathrm{CHs}$ are selected along with the main $\mathrm{CHs}$ to improve the life time. It is shown to perform better than original LEACH. However, SNs need extra energy for selecting vice CHs. In [16], E-LEACH protocol has been proposed; to address the residual energy consideration during selection of $\mathrm{CHs}$, which can extend the life of the network. A framework for energy estimation in cluster based protocol in WSNs has been proposed in [17]. A plenty of biologically inspired approaches clustering algorithms were also developed to extend the life time of the WSN. Centralized LEACH [18] was applied by simulated annealing. In $\mathrm{LEACH}-\mathrm{C}$ the selection of $\mathrm{CH}$ is done by $\mathrm{BS}$ and hence performs better. The intra cluster distance and energy of nodes are considered in LEACH-C and therefore, it can efficiently select the $\mathrm{CHs}$ to extend the lifetime of the network. Besides, the formation of clusters is ignored, which may lead to energy inefficiency of the network. In [19], PSO approach has been proposed to select the optimal location of CHs. However, the main objective of this algorithm was to reduce the intra-cluster distance, it ignores the distance to the sink, which is one of the desirable metric to improve the energy efficiency of the network for the direct communication of data to the sink. Also, non- $\mathrm{CH}$ nodes assigned to the $\mathrm{CHs}$ based on distance only, which may cause energy imbalance of the network. In [20], a PSO based cluster formation using altered fitness function includes both the intra-cluster distance and the sink distance has been proposed. However, the residual energy of the SNs are not taken care off. An energy aware $\mathrm{CH}$ selection using PSO-C is proposed in [21] by considering average intra-cluster distance and ratio of total initial energy of all nodes to the total present energy of the all $\mathrm{CHs}$, however it does not include the sink distance as it play a crucial role in energy saving. The new fitness function based on intra cluster distance, residual energy and node density is proposed in [22] for $\mathrm{CH}$ selection in clustering using PSO, but the cluster formation phase is ignored, which may cause severe energy in efficiency to the network. In [23] the real time comparison of LEACH-C, fuzzy-C means and Harmony search algorithms have been implemented, however the re-clustering issue has not been addressed. The CSO algorithm has been applied to FIR filter design in [27]. The real time optimal $\mathrm{CH}$ selection using $\mathrm{CSO}$ is performed in [28], however the simulation comparison has not been presented.

\section{WSN SYSTEM MODEL}

\subsection{NETWORK MODEL}

The proposed network model of WSN has $\mathrm{N}$ sensor nodes deployed in a random area of $M \times M \mathrm{~m}^{2}$ and formed in to k clusters such that $c_{1}, c_{2}, \ldots, c_{k}$. Following are the standard points with respect to the proposed protocol development:

- Both SN and BS are stationary and have same initial energy.

- At start up BS can directly communicate with all the sensor nodes to exchange the initial information.

- The initial energy for all the nodes is same and the network is homogeneous.
- Each SN knows its own position, which means the intra cluster distance between nodes are determined based on the received signal strength [24].

- SNs use different transmission power level based on the distance to which data to be sent to the $\mathrm{CH}$ or BS.

- The communication field range is fixed.

\subsection{ENERGY MODEL}

The energy model used in this paper is same that of the one used in [3]. In this model, transmitter dissipates energy to run the radio electronics and the power amplifier. The receiver dissipates energy to run the radio electronics. The energy consumption of the node depends on the amount of the data and distance $d$ to be sent. In this model, energy consumption of a node is proportional to $d^{2}$ when the propagation distance $d$ is less than the threshold distance $d_{0}$; otherwise it is proportional to $d^{4}$. The total energy consumption of each node in the network for transmitting the $l$ bit data packet is given by the following equations.

$$
\begin{aligned}
& E_{T x}(l, d)=1 \times E_{\text {ele }}+1 \times E_{f s} \times d^{2}, \text { if } \& d<d_{0} \\
& E_{T x}(l, d)=1 \times E_{\text {ele }}+1 \times E_{m p} \times d^{4}, \text { if } \& d \geq d_{0}
\end{aligned}
$$

where, $E_{\text {ele }}$ the energy is dissipated per bit to run the transmitter or receiver circuit, amplification energy for free space model $e_{f s}$ and for multi-path model $e_{m p}$ depends on the transmitter amplifier model and $d_{0}=\sqrt{E_{f s} / E_{m p}}$ is the threshold transmission distance.

In the same way to receive $l$ bit of data the energy consumed by the receiver is given by

$$
E_{R x_{C H}}=l \times E_{\text {ele }}
$$

where, $E_{\text {ele }}$ depends on several factors such as digital coding, modulation, filtering and the signal spreading. Note that this is a simplified model and actual radio wave propagation is highly variable and difficult to model.

Similarly, the energy consumed by $\mathrm{CH}$ to receive data from the cluster is given in Eq.(4),

$$
E_{R x_{C H}}=l \times E_{\text {ele }}\left(\frac{N}{k}-1\right)
$$

Also the energy consumed by the $\mathrm{CH}$ to transmit the data to the BS after aggregating a $l$-bit data is given below in Eq.(5)

$$
E_{R x_{C H}}=l \times E_{d a}\left(\frac{N}{k}\right)+l \times E_{e l e}\left(\frac{N}{k}-1\right)+l \times E_{m p} \times d_{t o B S}^{4}
$$

where, $E_{d a}$ is energy consumption of a $\mathrm{CH}$ for data aggregation and $k$ is number of clusters.

The network parameters used in the simulation performance is given in Table.1.

Table.1. Network Parameters

\begin{tabular}{|c|c|}
\hline Parameter & Value \\
\hline Sensor Field & $100 \times 100$ sq.m \\
\hline BS location & $(100-200),(100-200)$ \\
\hline Number of SNs & 100 \\
\hline Energy of SNs & $1 \mathrm{~J}$ \\
\hline$E_{\text {ele }}$ & $50 \mathrm{~nJ} / \mathrm{bit}$ \\
\hline
\end{tabular}




\begin{tabular}{|c|c|}
\hline$E_{f s}$ & $10 \mathrm{pJ} / \mathrm{bit} / \mathrm{m}^{2}$ \\
\hline$E_{m p}$ & $0.0013 \mathrm{pJ} / \mathrm{bit} / \mathrm{m}^{4}$ \\
\hline$E_{d a}$ & $5 \mathrm{~nJ} / \mathrm{bit} / \mathrm{message}$ \\
\hline$d_{m a x}$ & $100 \mathrm{~m}$ \\
\hline$d_{0}$ & $25 \mathrm{~m}$ \\
\hline Packet length & $4000 \mathrm{bits}$ \\
\hline Size of message & $500 \mathrm{bits}$ \\
\hline
\end{tabular}

\section{CSO BASED CLUSTER PROTOCOL}

\subsection{CAT SWARM OPTIMIZATION}

Cat Swarm Optimization (CSO) is one of the new heuristic optimization algorithms based on swarm intelligence [24-26] from the behaviour of the cat. It consists of two sub models representing the behaviour of cats namely "seeking mode" and "tracing mode".

\section{- Seeking Mode:}

Create copies of cats and it stores the present position of the cat. Calculate the fitness value for all the coordinate points by Eq.(6). Select the coordinate points and replace with best candidate points $\left(P_{i}\right)$.

$$
P_{i}=\frac{\left|f_{i}-f_{b}\right|}{f_{\max }-f_{\min }}
$$

where, $f_{i}$ is current fitness and $f_{b}$ is best fitness. If the objective is to minimize the solution then $f_{b}=f_{\min }$ otherwise $f_{b}=f_{\max }$.

\section{- Tracing Mode:}

The cats in this sub model depict the prey tracking of cat's behaviour. Update the velocities for each cat direction as per Eq.(7). Check the velocity for in range and if not it has to be set equal to the maximum limit. Update the position of each cat according to Eq.(8)

$$
\begin{gathered}
v_{i d}=v_{i d}+r \times c\left(x_{\text {best }}-x_{i d}\right) \\
x_{i d}=x_{i d}+v_{i d}
\end{gathered}
$$

where, $x_{i d}$ - current position of the cat and $x_{\text {best }}$ - local best position of cat, $v_{i d}$ - velocity of the cat in an $M$-dimensional solution space, $r$ - random number (0-1) and $c$ - acceleration constant (0.5-2). The Fig. 2 represents the flow chart of CSO algorithms applied for cluster set up phase.

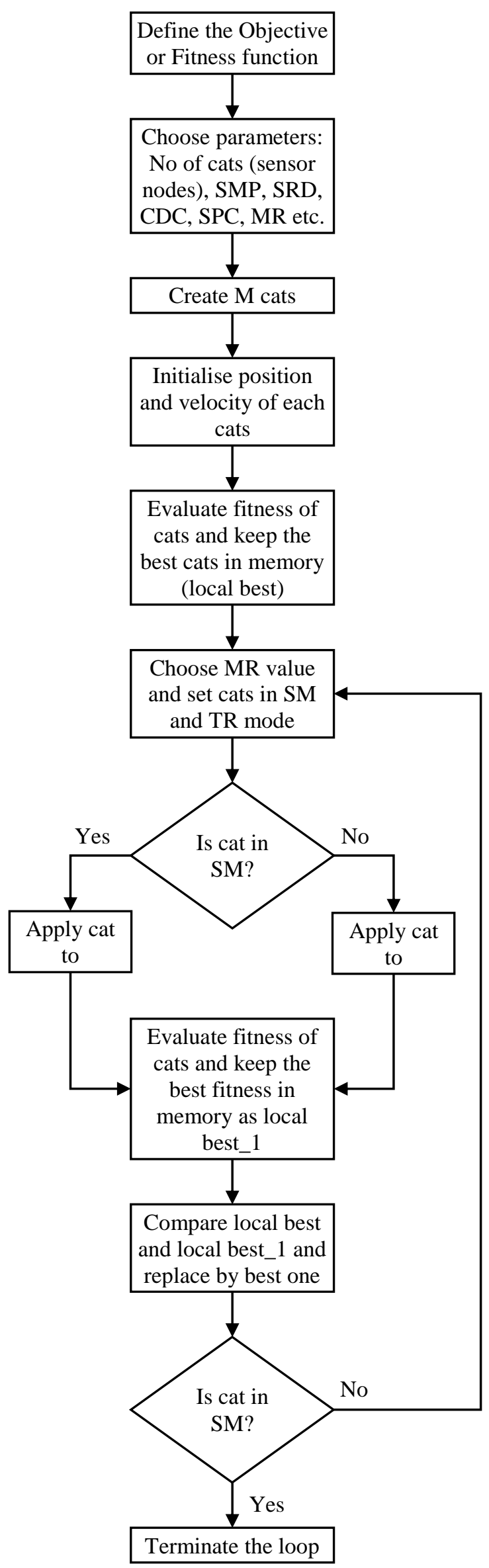

Fig.2. Flow chart of CSO algorithm for cluster setup phase 


\subsection{CLUSTER PROTOCOL AND FORMULATION OF OBJECTIVE FUNCTION}

The cluster protocol operation consists of two phases namely cluster setup and data transmission phase respectively.

Cluster Setup Phase: During this phase all the sensor nodes sends advertisement messages to the BS by including their position information and initial residual energy. Based on the information received from the SNS, the BS calculates the optimum $\mathrm{CHs}$ using among them by applying CSO algorithms and the same is forwarded to the sensor nodes about their $\mathrm{CHs}$ as advertisement (ADV) message. The process of setting up cluster is repeated periodically after the data transmission phase is completed. The optimum number of cluster $\left(c_{\text {opt }}\right)$ is formed [29] by using Eq.(9)

$$
C_{\text {opt }}=\sqrt{\frac{N}{2 \pi}} \sqrt{\frac{E_{f s}}{E_{m p}}} \sqrt{\frac{D_{n}}{d_{t o B S}^{2}}}
$$

where, $N$ is number of nodes, $D_{n}$ is network diameter, $d^{2}$ to BS is distance to base station. The Fig. 3 shows the number deployment of the sensor nodes and the $\mathrm{CH}$.

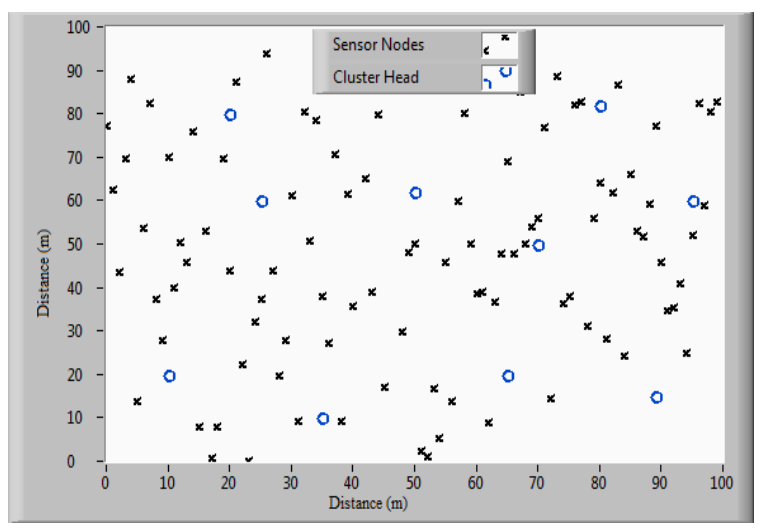

Fig.3. Clustering field in WSN

Data transmission Phase: In this phase a schedule has been initiated after all the SNs receives ADV message from the BS. Then the SNs sends their sensed data to their respective $\mathrm{CHs}$. The transmission power of the SNs are optimized since the minimal spatial distance to their $\mathrm{CHs}$. The radio of $\mathrm{CH}$ is turned $\mathrm{ON}$ comparatively long time to perform receiving information from its SNs, aggregating and forwarding the same to the BS. The main focus of the proposed CSO based cluster protocol is to select $\mathrm{CHs}$ amongst the sensor nodes in consideration with energy efficiency so as to increase the network lifetime. For the efficient $\mathrm{CH}$ selection with energy efficiency, we consider the intra cluster distance and residual energy of the sensor nodes including the $\mathrm{CH}$.

The optimization problem has to be formulated by considering the following:

- Intra cluster distance from sensor node to $\mathrm{CH}$ : In order to consume less energy, a sensor node $S N_{i}$ should only join a cluster head $\mathrm{CH}_{j}$ which is closest to $S N_{i}$ its transmission radius.

- $C H$ residual energy: A Sensor node $S N_{i}$ should only join a cluster head $\mathrm{CH}_{j}$ which has higher residual energy than other $\mathrm{CH}$ within the transmission radius.
Therefore the optimal $\mathrm{CH}$ selection problem can be mathematically defined as given in Eq.(10).

$$
\begin{gathered}
f_{o b j}=a \times f_{1}+(1-a) \times f_{2} \\
f_{1}=\max _{j \in(i, k)}\left\{\frac{\sum_{\forall S N_{i} \in c_{j}} d\left(S N_{i}, C H_{j}\right)}{\left|c_{j}\right|}\right\} \\
f_{2}=\sum_{j=1}^{k}\left\{\frac{\sum_{i=1}^{\left\|c_{k}\right\|} E_{i}^{r e s}}{E_{C H_{j}}^{r e s}}\right\}
\end{gathered}
$$

The objective function $f_{o b j}$ consist of two parts as indicated in the Eq.(10). The first part $f_{1}$ represents the maximum total Euclidean distance between the sensors nodes $\forall S N_{i}$ belongs to cluster $C_{j}$ to their cluster heads $C H_{j}$ and $\left|C_{j}\right|$ is the number of SNs that belong to $C_{j}$. By minimizing $f_{1}$ it tends to minimize the intracluster mean distance between these sensor nodes and their respective $\mathrm{CHs} . f_{2}$ is the sum of the ratio of present residual energy of all alive nodes $\sum_{i=1}^{\left\|c_{k}\right\|} E_{i}^{\text {res }}$ in the network with the current energy level of the $\mathrm{CH}_{j}$, which is $E_{\mathrm{CH}_{j}}^{r e s}$. To minimize $f_{2}$ the term $\sum_{i=1}^{\left\|c_{j}\right\|} E_{i}$ has to be minimal and $\Delta E_{C_{j}}$ must be maximized. Thus by minimizing the objective function $f_{o b j}$, the cluster formation and $\mathrm{CH}$ selection in WSN can be optimized and in turn the energy consumption also will be increased in the network. Minimizing $f_{2}$ yields optimal selection of $\mathrm{CH}$ to reduce the energy consumption in the network. The constant a represents the contribution of both $f_{1}$ and $f_{2}$ in the objective function.

\subsection{CSO ALGORITHM FOR CLUSTERING PROTOCOL}

The CSO program for the proposed cluster based protocol is written in LabVIEW using the following steps:

Step 1: Initialize all SNs with same value of energy and deploy them in $100 \times 100 \mathrm{~m}^{2}$ area.

Step 2: Assign 5\% of $N$ nodes as $\mathrm{CHs}$ in a random manner.

Step 3: Find the closest $\mathrm{CHs}$ for all the member nodes

Step 4: For $i$ : 1 to $N$ (Member nodes)

Step 5: Initialize min distance $d$ (distance of node $i$ from BS)

Step 6: For $j$ : 1 to $M$ (Number of $\mathrm{CHs}$ )

Step 7: Distance $=d_{i j}\left(\right.$ distance from $S N_{i}$ to $\left.C H_{j}\right)$

Step 8: If (min distance > distance)

Step 9: Min distance $=$ distance

Step 10: cluster $_{i d}=j$

Step 11: End if

Step 12: End for

Step 13: Determine the min average intra cluster distance and sink distance of all $\mathrm{CHs}\left(f_{1}\right)$ by Eq.(11)

Step 14: Compute $f_{2}$ to maximize the total energy of CHs using Eq.(12) 
Step 15: Calculate Fitness for each node

Step 16: For $i$ : 1 to $N$

Step 17:Fitness $(\mathrm{i})=a f_{1}+(1-a) f_{2}$

Step 18: Pbest $_{i}=$ Fitness $(i)$

Step 19: End for

Step 20: Initialize the velocity of each node $=0$.

Step 21: Assign the lowest value of Pbest $t_{i}$ as gbest and assign the value of Pbest $t_{i}$ as gbest

Step 22: For $i$ : 1 to total number of iterations

Step 23: Compute the position and velocity of each SNs using CSO algorithm by Eq.(5) and Eq.(6)

Step 24: Repeat steps 3-6 to find closest $\mathrm{CH}$ for all member nodes.

Step 25: Repeat step 7 and 8 for calculating $f_{1}$ and $f_{2}$.

Step 26: Calculate Fitness and update Pbest

Step 27: For $i$ : 1 to $N$

Step 28: If (Fitness $(i) \leq$ Pbest $\left.t_{i}\right)$

Step 29: Pbest $_{i}=$ Fitness $(i)$

Step 30: End if

Step 31: End For

Step 32: Repeat step 12 to compute gbest

Step 33: End For

The procedure for CSO based cluster protocol is represented as flow chart in Fig.4 and Fig.5. The Fig.4 shows the network operation performed at the BS and Fig.5 depicts the same in sensor nodes.

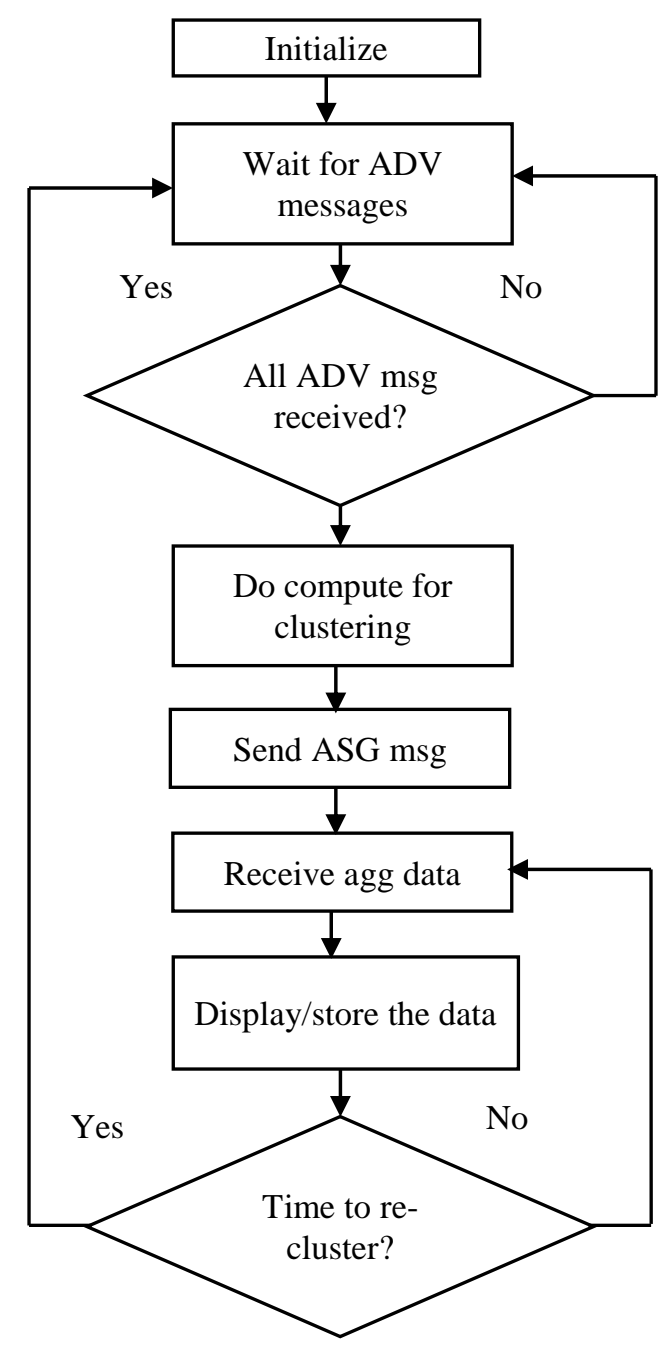

Fig.4. Flow chart of network operation at the BS

\section{RESULTS AND DISCUSSIONS}

The simulation performance is carried out using LabVIEW in windows based computer. The simulation parameters for LEACH-C have been referred from [18] and PSO from [21] and for CSO [30] as listed in Table.2.

Table.2. CSO Parameters

\begin{tabular}{|c|c|}
\hline Parameter & Value/Range \\
\hline$S M P$ & 5 \\
\hline$S R D$ & $20 \%$ \\
\hline$C D C$ & $80 \%$ \\
\hline$M R$ & 0.7 \\
\hline$c$ & 2 \\
\hline$r$ & 1 \\
\hline
\end{tabular}




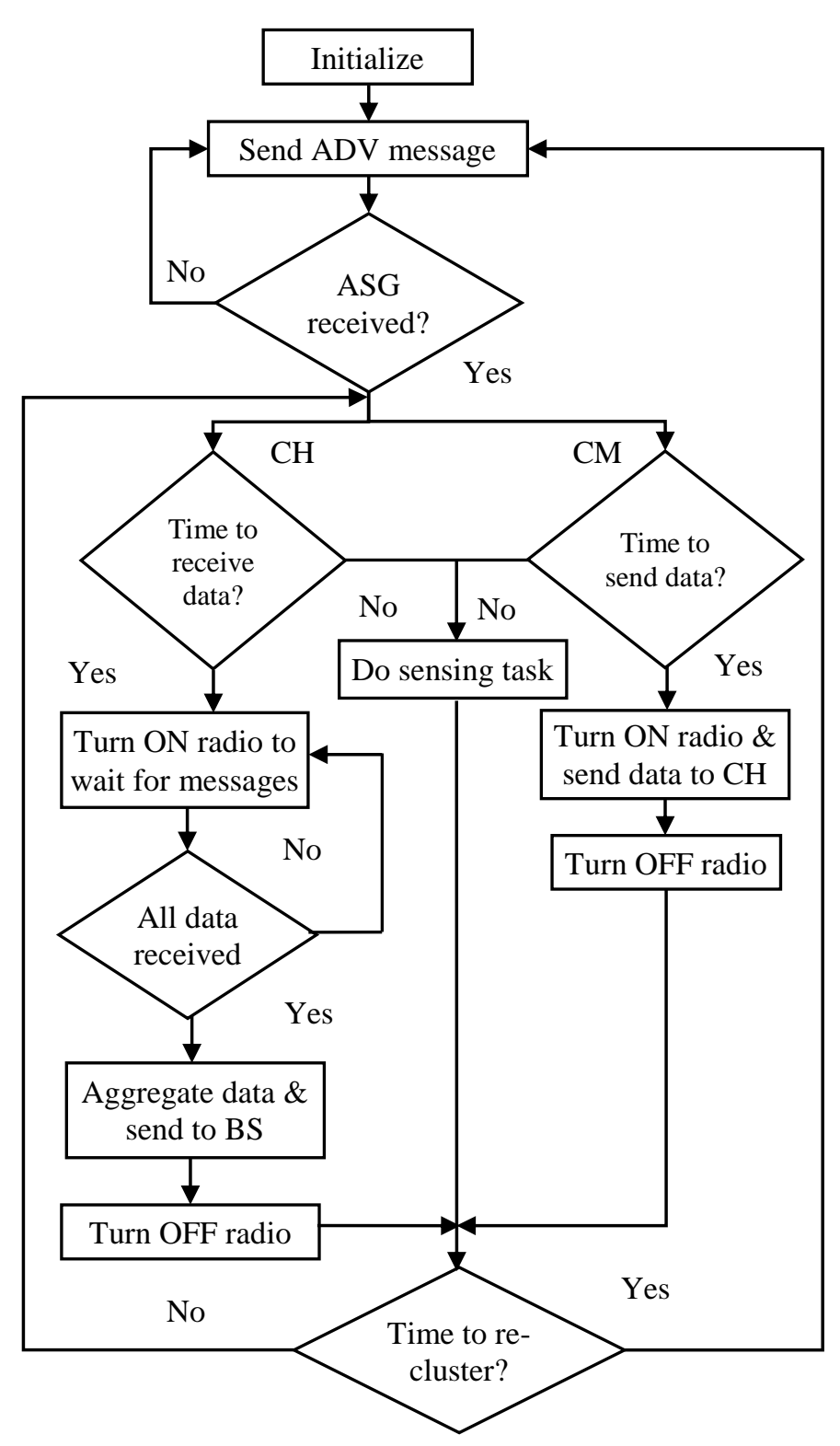

Fig.5. Flow chart of network operation at the SNs

In our proposed work, the BS station is positioned at the centre for the initial test run. While in consecutive test run we also varied the number of sensor nodes, percentage of possible cluster heads the position of the BS to verify the impact in performance. The algorithm is tested at initial population of 50 and varied up to 80 . The result is plotted as graphs by considering average of 25 times of algorithm run. The performance of the CSO based cluster protocol is measured by some important metrics namely fitness convergence, energy consumption, network life time and number of data received at the base station.

\subsection{COMPARISION OF CONVERGENCE}

The CSO has been applied to find the optimal $\mathrm{CHs}$ for the WSN. The well-known cluster protocol LEACH-C and evolutionary algorithm PSO also applied to solve the formulated optimization problem in order to compare the performance of CSO. The fitness convergence for the objective function as per Eq.(10) with the number of generations using LEACH-C, PSO and CSO are shown in Fig.6. In Fig.6, it can be seen that CSO converges at much faster rate to achieve comparatively better minimum fitness value for the objective function than $\mathrm{LEACH}-\mathrm{C}$ and PSO algorithm. The main drawback of PSO is its incapability to maintain the anticipated levels of population diversity and the balance between global and local searches. Hence the sub-optimal solutions for PSO is prematurely obtained. Also the PSO algorithm contains some tuning parameters which greatly influence the algorithm performance. In CSO the global and local search has different strategy. The velocity and position update is used only for the global search cats in tracing mode and not for the local search cats in seeking mode. This strategies helps to achieve faster convergence by CSO.

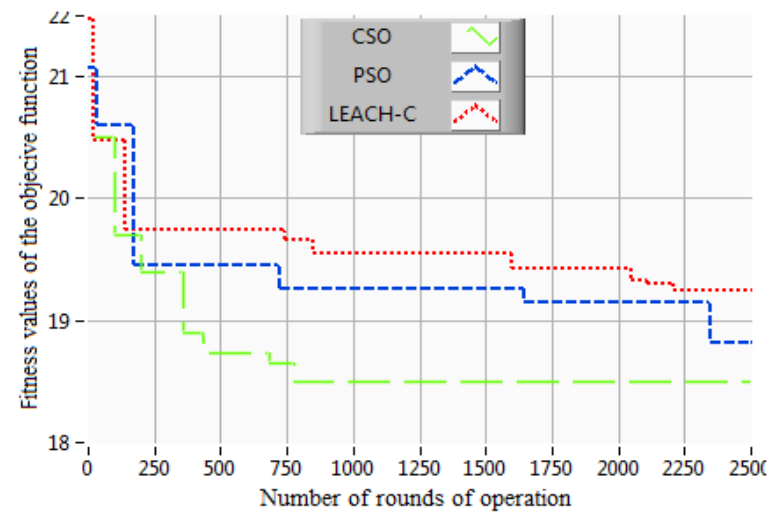

Fig.6. Fitness function convergence

\subsection{FINDING ON ENERGY CONSUMPTION AND NETWORK LIFETIME}

The prime parameter in energy consumption is residual energy of the node. As the number of rounds of operation increases the residual energy of SNs is dropping down, the proper selection of $\mathrm{CHs}$ plays a very vital role in reducing the energy consumption and the proposed algorithm play a suitable role in selecting the $\mathrm{CHs}$ using the fitness function. The energy consumption of the network over the number of rounds of operation represented in Fig.7 shows that the proposed algorithm is better in performance due to the fact that CSO deals with the fitness function which considers the intra cluster distance from the $\mathrm{CHs}$ to determine their optimal locations. The energy consumption of when applying CSO algorithms performs at least $19 \%$ over the PSO algorithm.

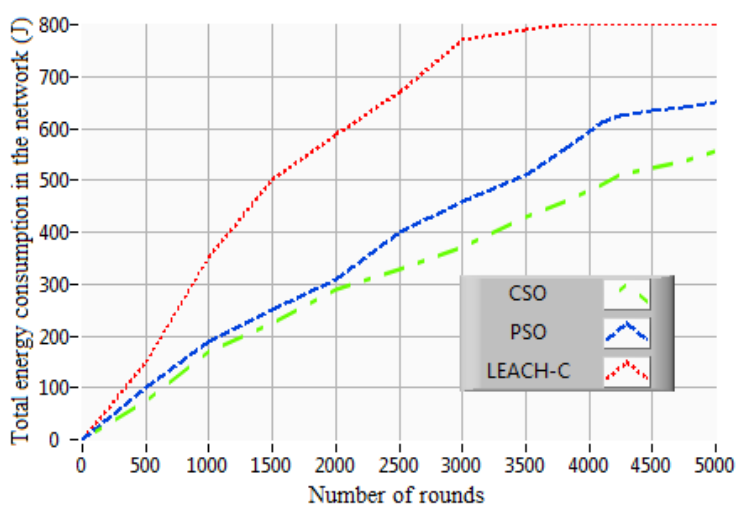

Fig.7. Number of rounds of operation vs. Energy consumption 
In our approach the $\mathrm{CH}$ is elected from other SNs with higher residual energy to increase the network lifetime. It can be noted from the Fig. 8 that the network lifetime of the proposed algorithm is better than other two algorithms. Also when the BS is kept at the centre of the field it is noted that it helps for better life time however, there is a decrease in the lifetime when it is positioned to the corner of the target field. The CSO algorithms produce better clustering set up of the network and the $\mathrm{CHs}$ are selected more efficiently across the network. The energy consumption of all nodes are reduced because of shorter distances between cluster members and their CHs. With CSO and PSO, the selection of $\mathrm{CH}$ is satisfied by using a cost function including SNs residual energy which helps to find the optimal $\mathrm{CH}$. However, with LEACH-Cthe clusters are created without considering residual energy, and $\mathrm{CHs}$ are chosen randomly among the SNs in the clusters. The Table. 3 depicts the summary of network life time parameters for the different protocols analysed in our approach.

Table.3. Network Lifetime

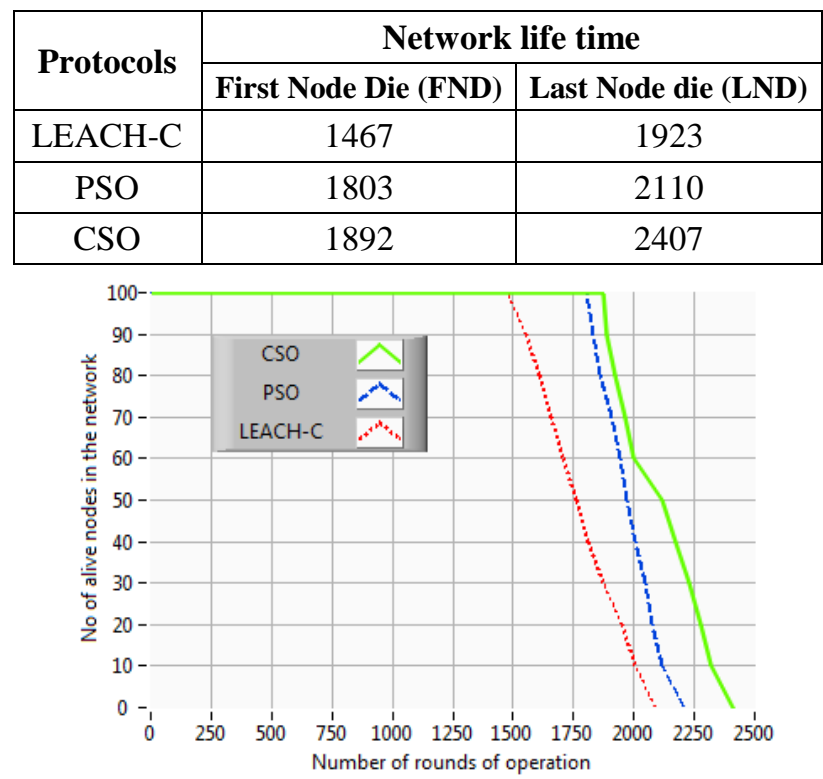

Fig.8. Network Lifetime

\subsection{FINDING ON DATA PACKET RECEIVED AT BASE STATION}

We also ran the CSO algorithm to compare the number of data packet received at the BS. As depicted in Fig.9, the proposed protocol receives higher number data at BS due to its better energy consumption and prolonging network lifetime compared to LEACH-C and PSO. It is also noted that when the BS location is at centre, the number of packets received is exceptionally more. Upon positioning the BS from the centre to the corner of the field, it is note that the number of data packets drops down. By the appropriate selection of the $\mathrm{CHs}$ with the help of efficient fitness function CSO outplays LEACH-C and PSO algorithms.

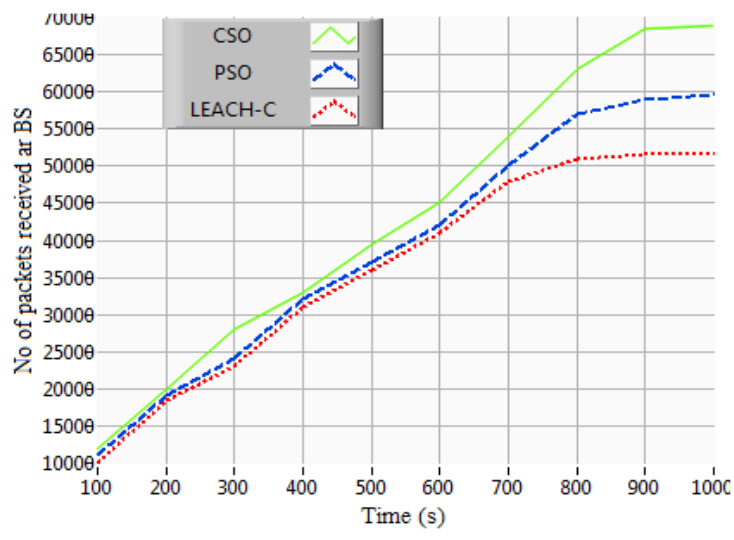

Fig.9. Time vs. Data received in BS

\section{CONCLUSION}

In this paper, an energy efficient clustering protocol has been proposed using CSO algorithm. The fast convergence of fitness function of CSO helps to choose proper cluster heads efficiently. We have also mostly focused on suitable formation of clusters through intra cluster distance, appropriate BS position, the residual energy of nodes to increase the efficiency of the network. The simulated results are evident that the proposed CSO based cluster protocol performs better than LEACH-C and PSO in terms of energy consumption, network lifetime, and the number of data packets received at the BS. The future work could focus to implement PSO-CSO hybridization in order to combine the global search method of the PSO with the local search method of the CSO. Another possible future direction may be adding a routing algorithm in addition to the proposed cluster protocol. Also the future work can try with heterogeneous network with different level of initial energy of sensor nodes.

\section{REFERENCES}

[1] Ian F.Akyildiz, Weilian Su, Yogesh Sankarasubramaniam and Erdal Cayirci, "A Survey on Sensor Networks", IEEE Communications Magazine, Vol. 40, No. 8, pp. 102-114, 2002.

[2] Kemal Akkaya and Mohamed Younis, "A Survey on Routing Protocols for Wireless Sensor Networks", Ad Hoc Networks, Vol. 3, No. 3, pp. 325-349, 2005.

[3] W.B. Heinzelman et al., "Energy Efficient Communication Protocol for Wireless Micro Sensor Networks", Proceedings of $33^{\text {rd }}$ Hawaii International Conference on System Sciences, pp. 112-117, 2000.

[4] X.Y. Liu et al., "CDC: Compressive Data Collection for Wireless Sensor Networks", IEEE Transactions on Parallel and Distributed Systems, Vol. 26, No. 8, pp. 2188-2197, 2015.

[5] L. Xiang et al., "Compressed Data Aggregation for Energy Efficient Wireless Sensor Networks", Proceedings of $8^{\text {th }}$ IEEE Communications Society Conference on Sensor, Mesh and Adhoc Communications and Networks, pp. 46-54, 2011.

[6] $\mathrm{X}$. Xu et al., "Hierarchical Data Aggregation using Compressive Sensing (HDACS) in WSNs", ACM Transactions on Sensor Networks, Vol. 11, No. 3, pp. 45-59, 2015. 
[7] O. Younis and S. Fahmy, "HEED: Hybrid Energy Efficient Distributed Clustering Approach for Ad Hoc Sensor Networks", IEEE Transactions on Mobile Computing, Vol. 3, No. 4, pp. 366-379, 2004.

[8] S. Lindsey and C.S. Raghavendra, "PEGASIS: Power Efficient Gathering in Sensor Information Systems", Proceedings of IEEE International Aerospace Conference, Vol. 3, pp. 1125-1130, 2002.

[9] Y. Yanjun. Qing Cao and Athanasios V. Vasilakos, "EDAL: An Energy-Efficient, Delay Aware, and Lifetime-Balancing Data Collection Protocol for Heterogeneous Wireless Sensor Networks", IEEE ACM Transactions on Networking, Vol. 23, No. 3, pp. 810-823, 2015.

[10] Y. Yanjun. Qing Cao and Athanasios V. Vasilakos, "EDAL: An Energy Efficient, Delay-Aware and Lifetime-Balancing Data Collection Protocol for Wireless Sensor Networks", Proceedings of $10^{\text {th }}$ International Conference on Mobile Ad hoc and Sensor Systems, pp. 182-190, 2013.

[11] G. Wei et al., "Prediction-based Data Aggregation in Wireless Sensor Networks: Combining Grey Model and Kalman Filter", Computer Communications, Vol. 34, No. 6, pp. 793-802, 2011.

[12] K. Han, "Algorithm Design for Data Communications in Duty Cycled Wireless Sensor Networks: A Survey", IEEE Communications Magazine, Vol. 51, No. 7, pp. 107-113, 2013.

[13] V. Loscri, "A Two-Level Hierarchy for Low-Energy Adaptive Clustering Hierarchy (TL-LEACH)", Proceedings of IEEE International Conference on Vehicular Technology, Vol. 62, No. 3, pp. 1809-1815, 2005.

[14] M. Xiaoyan, "Study and Design on Clustering Routing Protocols of Wireless Sensor Networks", Ph.D Dissertation, Department of Control Science and Engineering, Zhejiang University, 2006.

[15] M.B. Yassein, "Improvement of LEACH Protocol of Wireless Sensor Networks (VLEACH)", International Journal of Digital Content Technologies and Applications, Vol. 3, No. 2, pp. 132-136, 2009.

[16] X. Fan and S. Yulin, "Improvement on LEACH Protocol of Wireless Sensor Network", Proceedings of International Conference on Sensor Technologies and Applications, pp. 260-264, 2007.

[17] N. Zhu and A.V. Vasilakos, "A Generic Framework for Energy Evaluation on Wireless Sensor Networks", Wireless Networks, Vol. 22, No. 4, pp. 1199-1220, 2015.

[18] W.B. Heinzelman, A.P. Chandrakasan and H. Balakrishnan, "An Application Specific Protocol Architecture for Wireless Microsensor Networks", IEEE Transactions on Wireless Communications, Vol. 1, No. 4, pp. 660-670, 2002.

[19] J. Tillet, "Cluster Head Identification in Adhoc Sensor Networks using Particle Swarm Optimization”, Proceedings of IEEE International Conference on Personal Wireless Communications, pp. 201-205, 2002.

[20] S.M. Guru, S.K. Halgamuge and S. Fernando, "Particle Swarm Optimisers for Cluster Formation in Wireless Sensor Networks", Proceedings of International Conference on Intelligent Sensors Sensor Networks and Information Processing, pp. 319-324, 2005.

[21] N.M.A. Latiff, C.C. Tsimenidis and B.S. Sharif, "EnergyAware Clustering for Wireless Sensor Networks using Particle Swarm Optimization", Proceedings of $18^{\text {th }}$ Annual IEEE International Symposium on Personal, Indoor and Mobile Radio Communications, pp. 1-5, 2007.

[22] Buddha Singh and Daya Krishan Lobiyal, "A Novel Energy Aware Cluster Head Selection based on Particle Swarm Optimization for Wireless Sensor Networks", HumanCentric Computing and Information Sciences, Vol. 2, No. 1, pp. 2-13, 2012.

[23] Duc Chinh Hoang, Parikshit Yadav, Rajesh Kumar and Sanjib Kumar Panda, "Real-Time Implementation of a Harmony Search Algorithm-based Clustering Protocol for Energy Efficient Wireless Sensor Networks", IEEE Transactions on Industrial Informatics, Vol. 10, No. 1, pp. 774-783, 2014.

[24] Shu-Chuan, Pei-Wei Tsai and Jeng-Shyang Pan, "Cat Swarm Optimization", Proceedings of Pacific Rim International Conference on Artificial Intelligence, pp. 854-858, 2006.

[25] Shu-Chuan and Pei-Wei Tsai, "Computational Intelligence based on the Behaviour of Cats", International Journal of Innovative Computing, Information and Control, Vol. 3, No. 1, pp. 163-173, 2007.

[26] Mahdi Bahrami, Omid Bozorg-Haddad and Xuefeng Chu, "Cat Swarm Optimization (CSO) Algorithm", Proceedings of International Conference on Advanced Optimization by Nature-Inspired Algorithms, pp. 9-17, 2016.

[27] Suman Kumar Saha, "Cat Swarm Optimization Algorithm for Optimal Linear Phase FIR Filter Design”, ISA Transactions, Vol. 52, No. 6, pp. 781-794, 2013.

[28] D. Chandirasekaran and T. Jayabarathi, "Cat Swarm Algorithm in Wireless Sensor Networks for Optimized Cluster Head Selection: A Real Time Approach", Cluster Computing, pp. 1-11, 2017.

[29] D.C. Hoang, R. Kumar and S.K. Panda, "Fuzzy C-means Clustering Protocol for Wireless Sensor Networks", Proceedings of IEEE International Symposium on Industrial Electronics, pp. 3477-3482, 2010.

[30] J. So and W.K. Jenkins, "Comparison of Cat Swarm Optimization with Particle Swarm Optimization for IIR System Identification”, Proceedings of IEEE International Conference on Signals, Systems and Computers, pp. 903910, 2013. 